\title{
Multimodal Emotion Recognition and Sentiment Analysis via Attention Enhanced Recurrent Model
}

\author{
Licai Sun* \\ School of Artificial Intelligence, \\ University of Chinese Academy of \\ Sciences \\ NLPR, Institute of Automation, \\ Chinese Academy of Sciences \\ sunlicai2019@ia.ac.cn \\ Bin Liu \\ NLPR, Institute of Automation, \\ Chinese Academy of Sciences \\ liubin@nlpr.ia.ac.cn
}

\author{
Mingyu $\mathrm{Xu}^{*}$ \\ NLPR, Institute of Automation, \\ Chinese Academy of Sciences \\ School of Artificial Intelligence, \\ University of Chinese Academy of \\ Sciences \\ xumingyu2021@ia.ac.cn \\ Jianhua Tao \\ NLPR, Institute of Automation, \\ Chinese Academy of Sciences \\ School of Artificial Intelligence, \\ University of Chinese Academy of \\ Sciences \\ CAS Center for Excellence in Brain \\ Science and Intelligence Technology \\ jhtao@nlpr.ia.ac.cn \\ Yuan Cheng \\ Ant Financial Services Group \\ chengyuan.c@antgroup.com
}

\author{
Zheng Lian \\ NLPR, Institute of Automation, \\ Chinese Academy of Sciences \\ lianzheng2016@ia.ac.cn
}

\begin{abstract}
With the proliferation of user-generated videos in online websites, it becomes particularly important to achieve automatic perception and understanding of human emotion/sentiment from these videos. In this paper, we present our solutions to the MuSe-Wilder and MuSe-Sent sub-challenges in MuSe 2021 Multimodal Sentiment Analysis Challenge. MuSe-Wilder focuses on continuous emotion (i.e., arousal and valence) recognition while the task of MuSe-Sent concentrates on discrete sentiment classification.

To this end, we first extract a variety of features from three common modalities (i.e., audio, visual, and text), including both low-level handcrafted features and high-level deep representations from supervised/unsupervised pre-trained models. Then, the long short-term memory recurrent neural network, as well as the selfattention mechanism is employed to model the complex temporal dependencies in the feature sequence. The concordance correlation coefficient (CCC) loss and F1-loss are used to guide continuous regression and discrete classification, respectively. To further
\end{abstract}

\footnotetext{
${ }^{*}$ Equal contribution.
}

Permission to make digital or hard copies of all or part of this work for personal or classroom use is granted without fee provided that copies are not made or distributed for profit or commercial advantage and that copies bear this notice and the full citation on the first page. Copyrights for components of this work owned by others than ACM must be honored. Abstracting with credit is permitted. To copy otherwise, or republish, to post on servers or to redistribute to lists, requires prior specific permission and/or a fee. Request permissions from permissions@acm.org.

MuSe '21, October 24, 2021, Virtual Event, China

(C) 2021 Association for Computing Machinery.

ACM ISBN 978-1-4503-8678-4/21/10 . \$ \$15.00

https://doi.org/10.1145/3475957.3484456 boost the model's performance, we adopt late fusion to exploit complementary information from different modalities. Our proposed method achieves CCCs of 0.4117 and 0.6649 for arousal and valence respectively on the test set of MuSe-Wilder, which outperforms the baseline system (i.e., 0.3386 and 0.5974 ) by a large margin. For MuSe-Sent, F1-scores of 0.3614 and 0.4451 for arousal and valence are obtained, which also outperforms the baseline system significantly (i.e., 0.3512 and 0.3291 ). With these promising results, we ranked top3 in both sub-challenges.

\section{CCS CONCEPTS}

- Information systems $\rightarrow$ Multimedia and multimodal retrieval; • Computing methodologies $\rightarrow$ Artificial intelligence.

\section{KEYWORDS}

Continuous Emotion Recognition; Discrete Sentiment Classification; Long Short-Term Memory; Self-Attention; Multimodal Fusion

\section{ACM Reference Format:}

Licai Sun, Mingyu Xu, Zheng Lian, Bin Liu, Jianhua Tao, Meng Wang, and Yuan Cheng. 2021. Multimodal Emotion Recognition and Sentiment Analysis via Attention Enhanced Recurrent Model. In Proceedings of the 2nd Multimodal Sentiment Analysis Challenge (MuSe '21), October 24, 2021, Virtual Event, China. ACM, New York, NY, USA, 6 pages. https://doi.org/10. $1145 / 3475957.3484456$

\section{INTRODUCTION}

Automatic emotion/sentiment recognition is a crucial component of natural human-machine interactions. It has a wide range of 
applications in modern scenarios, ranging from call-center dialogue systems to conversational agents.

There are two mainstream theories for emotion representation: the discrete emotion theory and the dimensional emotion theory [28]. As for the discrete emotion theory, a person's emotional state can be described by a few affective attributes (such as happy and sad). As for the dimensional emotion theory, it models the emotional state in a continuous space described by several dimensions (such as arousal, valence, and dominance). Thus, compared with the discrete emotion theory, the dimensional emotion theory describes more subtle and complicated emotional behaviors.

The MuSe 2021 Multimodal Sentiment Analysis Challenge [23] adopts the dimensional theory for emotion representation. In the MuSe-Wilder sub-challenge, the task is to predict continuous dimensional values of arousal and valence from three common modalities (i.e., audio, visual, and text). This sub-challenge is an extension of MuSe-Wild 2020. Note that this year's gold standard annotation is based on a completely novel continuous annotator fusion technique. Thus, although the video data is the same, the emotion annotations for arousal and valence are different from those in the last year. In the MuSe-Sent sub-challenge, the task is simplified to predict five advanced sentiment classes on a segment level. These discrete classes are created from continuous annotations of MuSe-Wilder through the extraction of time-series features and the application of unsupervised clustering. Please refer to the baseline paper [23] for more details.

In this paper, we present our solutions to the MuSe-Wilder and MuSe-Sent sub-challenges in MuSe 2021. Since good features are essential to robust emotion recognition, we first explore a series of features from the audio, visual, and text modality, including both low-level handcrafted features and high-level deep representations from supervised/unsupervised pre-trained models. For the temporal model, we utilize the long short-term memory (LSTM) recurrent neural network as well as the self-attention mechanism to capture complex temporal dynamics in the feature sequence. We hypothesize that the combination of LSTM and self-attention mechanism could enhance the model's ability of long-term temporal context modeling and is more suitable for continuous emotion recognition and discrete sentiment classification. To boost the model's performance, we adopt a simple yet effective fusion method, i.e., late fusion, to integrate information from different modalities. Since the discrete sentiment classes in MuSe-Sent are derived from continuous emotion annotations in MuSe-Wilder, we also exploit their relations to improve the performance of sentiment classification in MuSe-Sent. Experimental results show that our proposed method achieves significantly better performance than the baseline system for arousal and valence in both MuSe-Wilder and MuSe-Sent. With these encouraging results, we ranked top3 among all participants in both sub-challenges.

\section{METHOD}

The MuSe-Wilder sub-challenge requires participants to predict emotional dimensions (i.e., arousal and valence) in a time-continuous manner (i.e., every $0.25 \mathrm{~s}$ ), while the task of MuSe-Sent is to recognize five advanced sentiment classes on a segment level. Our proposed method mainly consists of four modules: feature extraction module, temporal model, loss function, and fusion module. The feature extraction module extracts a variety of features from the audio, visual, and text modality, including both low-level handcrafted features and high-level deep representations from supervised/unsupervised pre-trained models. Then, the temporal model is used to capture the complex temporal dependencies in the feature sequence. To guide the model training, the concordance correlation coefficient (CCC) loss and F1-loss are employed for MuSe-Wilder and MuSe-Sent, respectively. Finally, we adopt a late fusion module to integrate multimodal information to further boost the system's performance. In the following sections, we elaborate on these modules one by one.

\subsection{Feature Extraction}

Audio Features. We employ the classic eGeMAPS [7] feature and IS13 [22] feature as low-level handcrafted acoustic features. For eGeMAPS, we adopt the feature provided by the organizers of MuSe 2021. For IS13, we use the openSMILE toolkit [8] to extract it. Additionally, we employ the PyAudioAnalysis library [10] to extract a 34-dimensional feature vector (termed as PyAudio feature), which includes zero-crossing rate, spectral spread, and chroma vector. Note that IS13 and PyAudio features are extracted with a window size of $0.025 \mathrm{~s}$ and a hop size of $0.01 \mathrm{~s}$. To align with the label interval (i.e., $0.25 \mathrm{~s}$ ), we take the average feature around each label timestamp. For the high-level deep acoustic representations, we utilize several supervised/unsupervised models pre-trained on large-scale audio datasets, including VGGish [12], PANNs [14], and wav2vec 2.0 [2]. VGGish and PANNs are trained on the AudioSet dataset [9] for general audio classification, while wav2vec 2.0 is a framework for self-supervised speech representation learning. For PANNs, we use a CNN14 system trained with $16 \mathrm{kHz}$ audio recordings. For wav2vec 2.0, we use a base model trained on the Librispeech corpus [19]. All high-level acoustic features are extracted with a window size of about $1 \mathrm{~s}$ and a hop size of $0.25 \mathrm{~s}$.

Visual Features. We employ the facial action units (FAUs) feature provided by the organizers as the low-level handcrafted visual features. For the high-level visual representations, we utilize several deep neutral networks trained on large-scale face or facial expression datasets, including VGGFace [20], ResNetFace [1], SENetFace [14], and EmoFAN [26]. VGGFace is a face recognition model, which aims to extract general facial features from faces. For VGGFace, we use the feature provided by the organizers. ResNetFace and SENetFace are first pre-trained on a large-scale face recognition dataset and then fine-tuned on a commonly used facial expression dataset, FER+ [4]. The accuracy on the test set of FER+ is $87.4 \%$ and $88.9 \%$, respectively. For EmoFAN, it is a custom deep neural network trained on AffectNet [18] to analyze facial affect in naturalistic conditions. To extract these high-level visual representations, we first use the OpenFace toolkit [3] to obtain aligned faces from raw videos. Then, the face for each label timestamp is fed into the above models and the embedding before the fully connected layer is extracted. For those timestamps with no face, zero paddings are used.

Text Features. Transformer-based language models pre-trained on large-scale text corpora in an unsupervised manner have achieved 
extraordinary performance on many downstream tasks in natural language processing. Thus, we employ several state-of-the-art language models to derive high-level deep contextual word embeddings, including BERT, [6], RoBERTa [17], ALBERT [15], ELECTRA [5], and DeBERTa [11]. Among these models, BERT is a pioneering work of bidirectional encoder representations from Transformers. RoBERTa builds on BERT and introduces several modifications for more robust pretraining. ALBERT is a lite BERT by utilizing parameter reduction techniques for lower memory consumption and increasing the model training speed. ELECTRA employs a sampleefficient pre-training task and trains the model in a generative adversarial manner. DeBERTa improves BERT and RoBERTa using the disentangled attention mechanism and an enhanced mask decoder. For these BERT-like models, we use their large version (also uncased version if exists) and calculate the sum of outputs from the last four layers as final features.

\subsection{Temporal Model}

Our temporal model is made up of the self-attention layer, LSTM layer, and fully connected layer. As one of the state-of-the-art temporal analysis models, we use bidirectional LSTM as the main module to capture long-term temporal dependencies in the feature sequence. However, LSTM could not handle very long sequences, which is common in continuous emotion recognition. Since the self-attention mechanism can relate two different positions without regard to their distance in the sequence [27], following [25], we add self-attention before LSTM for better temporal context modeling. Finally, the fully connected layer maps the contextual features to continuous values or discrete classes. Suppose the input feature sequence is $X=\left\{x_{1}, x_{2}, \ldots, x_{T}\right\}$, the whole process can be summarized as follows:

$$
\begin{aligned}
H & =\operatorname{SelfAttention}(X) \\
H & =\operatorname{BiLSTM}(H) \\
\hat{Y} & =F C(H)
\end{aligned}
$$

where $\hat{Y}=\left\{\hat{y}_{1}, \hat{y}_{2}, \ldots, \hat{y}_{T}\right\}$ is the prediction.

\subsection{Loss Function}

As suggested by the last year's winner [25] in MuSe-Wilder, the concordance correlation coefficient (CCC) [16] loss generally outperforms either mean squared loss or L1 loss, therefore, we employ the CCC loss as the loss function for this sub-challenge. It is defined as follows:

$$
\begin{aligned}
\mathcal{L}_{c c c} & =1-c c c \\
c c c & =\frac{2 \rho \sigma_{\hat{Y}} \sigma_{Y}}{\sigma_{\hat{Y}}^{2}+\sigma_{Y}^{2}+\left(\mu_{\hat{Y}}-\mu_{Y}\right)^{2}}
\end{aligned}
$$

where $\mu_{\hat{Y}}$ and $\mu_{Y}$ are the mean of the prediction $\hat{Y}$ and the label $Y$, respectively. $\sigma_{\hat{Y}}$ and $\sigma_{Y}$ are their standard deviations. $\rho$ is the Pearson correlation coefficient (PCC) between $\hat{Y}$ and $Y$.

For the MuSe-Sent sub-challenge, we employ F1-loss as the loss function. Empirically, we find that F1-loss usually performs better than the standard cross-entropy loss. The F1 loss is calculated as follows:

$$
\begin{aligned}
\mathcal{L}_{f 1} & =1-f 1 \\
T P & =\sum_{i=1}^{N} \hat{Y}_{i} \odot Y_{i} \\
F P & =\sum_{i=1}^{N}\left(1-\hat{Y}_{i}\right) \odot Y_{i} \\
F N & =\sum_{i=1}^{N} \hat{Y}_{i} \odot\left(1-Y_{i}\right) \\
F 1 & =\frac{2 T P}{2 T P+F P+F N} \\
f 1 & =\mu_{F 1}
\end{aligned}
$$

where $\hat{Y}_{i}$ and $Y_{i}$ is the prediction and label for the $i$ th segment, $\odot$ denotes the Hadamard product, $N$ is the number of segments in one training batch, $\mu_{F 1}$ is the mean of $\mathrm{F} 1$ over five discrete sentiment classes.

\subsection{Fusion Method}

Early fusion and late fusion are two common methods for multimodal fusion. Although late fusion ignores cross-modal feature interactions, we empirically find that it works better than early fusion, which is also verified in [25]. We believe that this might be caused by the curse of high dimensionality in early fusion. Therefore, in this paper, we adopt late fusion for continuous emotion recognition and discrete sentiment classification. For the fusion model, we employ a second-level bidirectional LSTM to fuse the predictions from several unimodal predictions. Suppose that there are $M$ unimodal predictions $\left\{\hat{Y}^{m}\right\}_{m=1}^{M}$, the late fusion can be summarized as follows:

$$
\begin{aligned}
X & =\operatorname{Concat}\left(\hat{Y}^{1}, \hat{Y}^{2}, \ldots, \hat{Y}^{M}\right) \\
H & =\operatorname{BiLSTM}(X) \\
\hat{Y} & =F C(H)
\end{aligned}
$$

where $\hat{Y}$ is the final multimodal prediction.

\section{EXPERIMENTS}

\subsection{Dataset}

The MuSe-CaR [24] dataset is used for the MuSe-Wilder and MuSeSent sub-challenges in MuSe 2021 [23]. It is a large multimodal (audio, viusal, and text) car review dataset, which consists of 291 videos of 70 host speakers collected from YouTube. Note that several in-the-wild characteristics exist in the MuSe-CaR dataset. For example, 1) extremely non-frontal face angles, face occlusion, missing faces, and highly varying backgrounds in videos; 2) ambient noises in audio recordings; 3 ) usage of colloquial words and domainspecific terms in speaker utterances. These characteristics make performing continuous emotion recognition or discrete sentiment classification very challenging.

For the MuSe-Wilder sub-challenge, a total $35 \mathrm{~h}: 08 \mathrm{~m}: 01 \mathrm{~s}$ of video data is annotated per $0.25 \mathrm{~s}$ for arousal and valence. The numbers of videos in the training, validation, and test sets are 165,62 , and 64 , respectively. The evaluation metric for MuSe-Wilder is CCC, which 
is defined in equation (14). For MuSe-Sent, a total 35h:39m:49s of video data is annotated on a segment level for arousal and valence. The evaluation metric for this sub-challenge is the macro F1-score. The readers can refer to the baseline paper [23] for more details.

\subsection{Experimental Setup}

Data Preprocessing: Since the annotation for MuSe-Wilder is dense (i.e., 4 frames per second), we follow [25] and split each video into multiple segments with a window size of 200 frames (50s) and a hop size of 100 frames (25s). We emprically find that the video segmentation not only enriches training samples but also facilitate model convergence. Besides, we apply Z-score normalization to handcrafted acoustic features (i.e., eGeMAPS, IS13, and PyAudio feature) before feeding them into the temporal model.

Model Training: We implement our models using the PyTorch toolkit [21]. Specifically, for the temporal model, we use one selfattention layer and one bidirectional LSTM layer. The number of hidden neurons in the model is 64 for arousal and 128 for valence. Four heads are used in the self-attention layer. We employ an Adam optimizer [13] with a learning rate of 0.005 and a batch size of 1024 to train the model for a maximum number of 100 epochs. We halve the learning rate if the training loss cannot be optimized for five consecutive epochs. For the late fusion model, a bidirectional LSTM with 32 hidden neurons is employed to fuse several unimodal predictions. We train the fusion model using an Adam optimizer with a learning rate of 0.001 and a batch size of 64 for a maximum number of 30 epochs.

\subsection{Unimodal Results}

We first conduct a series of unimodal experiments on the validation set of two sub-challenges to investigate the effectiveness of low-level handcrafted features and high-level deep representations extracted from each modality.

The results of MuSe-Wilder are shown in Table 1. We can find that: 1) Audio features usually are more effective than visual and text features on arousal. 2) Text modality performs significantly better than the other two modalities on valence. 3) The visual modality generally achieves the worst result. These observations are consistent with previous results [23, 25]. For 1) and 2), we believe that the reason is that the perception of arousal is mainly dependent on how people speak (i.e., intonation and tone, etc.) while the perception of valence is mostly reflected by what people speak. For 3), we suggest that highly in-the-wild factors (such as extremely non-frontal face angles and missing face) in videos make performing continuous emotion recognition from the visual modality more challenging than audio and text modalities. For MuSe-Wilder, the best unimodal results for arousal and valence are achieved by wav2vec and DeBERTa, which are 0.4618 and 0.5249 respectively.

For MuSe-Sent, the unimodal results are shown in Table 2. We notice that the difference between different modalities is smaller than MuSe-Wilder. Besides, some observations in MuSe-Wilder do not hold in this sub-challenge. For example, although the five sentiment classes of MuSe-Sent derive from continuous emotion annotations of MuSe-Wilder, text modality does not perform best for valence classification. We suggest that mapping continuous annotations to discrete classes might lose too much information,
Table 1: Unimodal results on the validation set of MuSeWilder. " $A$ ", " $V$ ", and " $T$ " denote the audio, visual, and text modality, respectively.

\begin{tabular}{c|c|c|c|c}
\hline Feature & Modality & Dimension & Arousal & Valence \\
\hline eGeMAPS & $\mathrm{A}$ & 88 & 0.3784 & 0.1693 \\
PyAudio & $\mathrm{A}$ & 34 & 0.3596 & 0.1884 \\
IS13 & $\mathrm{A}$ & 130 & 0.3850 & 0.1555 \\
VGGish & $\mathrm{A}$ & 128 & 0.4213 & 0.1756 \\
CNN14 & $\mathrm{A}$ & 2048 & 0.4608 & 0.2017 \\
wav2vec & $\mathrm{A}$ & 768 & $\mathbf{0 . 4 6 1 8}$ & 0.2225 \\
\hline FAUs & $\mathrm{V}$ & 35 & 0.2926 & 0.1039 \\
VGGFace & $\mathrm{V}$ & 512 & 0.3016 & 0.1141 \\
EmoFAN & $\mathrm{V}$ & 256 & 0.3682 & 0.0506 \\
SENetFace & $\mathrm{V}$ & 2048 & 0.3684 & 0.0615 \\
ResNetFace & $\mathrm{V}$ & 2048 & 0.3891 & 0.0775 \\
\hline BERT & $\mathrm{T}$ & 1024 & 0.3512 & 0.4518 \\
RoBERTa & $\mathrm{T}$ & 1024 & 0.3583 & 0.5143 \\
ALBERT & $\mathrm{T}$ & 1024 & 0.3672 & 0.4414 \\
ELECTRA & $\mathrm{T}$ & 1024 & 0.3656 & 0.4735 \\
DeBERTa & $\mathrm{T}$ & 1024 & 0.3832 & $\mathbf{0 . 5 1 8 1}$ \\
\hline
\end{tabular}

Table 2: Unimodal results on the validation set of MuSe-Sent. " $A$ ", " $V$ ", and " $T$ " denote the audio, visual, and text modality, respectively.

\begin{tabular}{c|c|c|c|c}
\hline Feature & Modality & Dimension & Arousal & Valence \\
\hline eGeMAPS & $\mathrm{A}$ & 88 & 0.3211 & 0.3271 \\
PyAudio & $\mathrm{A}$ & 34 & 0.2514 & 0.2334 \\
IS13 & $\mathrm{A}$ & 130 & 0.2619 & 0.2284 \\
VGGish & $\mathrm{A}$ & 128 & 0.2117 & $\mathbf{0 . 3 3 5 8}$ \\
CNN14 & $\mathrm{A}$ & 2048 & $\mathbf{0 . 3 8 0 4}$ & 0.3106 \\
Wav2vec & $\mathrm{A}$ & 768 & 0.2304 & 0.2729 \\
\hline FAUs & $\mathrm{V}$ & 35 & 0.3444 & 0.3017 \\
VGGFace & $\mathrm{V}$ & 512 & 0.2623 & 0.3032 \\
EmoFAN & $\mathrm{V}$ & 256 & 0.3327 & 0.2784 \\
SENetFace & $\mathrm{V}$ & 2048 & 0.2607 & 0.2836 \\
ResNetFace & $\mathrm{V}$ & 2048 & 0.2546 & 0.2909 \\
\hline BERT & $\mathrm{T}$ & 1024 & 0.3145 & 0.3149 \\
RoBERTa & $\mathrm{T}$ & 1024 & 0.3097 & 0.3106 \\
ALBERT & $\mathrm{T}$ & 1024 & 0.3124 & 0.3037 \\
ELECTRA & $\mathrm{T}$ & 1024 & 0.3121 & 0.2889 \\
DeBERTa & $\mathrm{T}$ & 1024 & 0.3402 & 0.3266 \\
\hline
\end{tabular}

thus makes directly performing valence classification from the text modality too difficult. Finally, two audio features, CNN14 and VGGish, achieve the best unimodal results for arousal and valence in MuSe-Sent, which are 0.3804 and 0.3358 respectively.

\subsection{Multimodal Results}

In this section, we present several combinations of top-performing features from different modalities to investigate the effectiveness of late fusion. The multimodal results on the validation set of MuSeWilder of MuSe-Sent are shown in Table 3. We can observe that late fusion significantly boost the model's performance for both arousal 
Table 3: Multimodal results on the validation set of MuSeWilder of MuSe-Sent. "Wilder Arousal" and "Wilder Valence" means the best multimodal predictions from MuSe-Wilder.

\begin{tabular}{c|c|c|c}
\hline Task & Features & Arousal & Valence \\
\hline \multirow{5}{*}{ MuSe-Wilder } & $\begin{array}{c}\text { IS13+CNN14+ } \\
\text { wav2vec+EmoFAN+ } \\
\text { ResNetFace+DeBERTa }\end{array}$ & 0.5549 & - \\
\cline { 2 - 4 } MuSe-Sent & $\begin{array}{c}\text { CNN14+wav2vec+ } \\
\text { VGGFace+FAUs+ } \\
\text { RoBERTa+DeBERTa }\end{array}$ & - & 0.5566 \\
\cline { 2 - 4 } & $\begin{array}{c}\text { CNN14+FAUs } \\
\text { FAUs+Wilder Arousal }\end{array}$ & 0.4117 & - \\
\cline { 2 - 4 } & $\begin{array}{c}\text { VGGish+VGGFace+ } \\
\text { DeBERTa }\end{array}$ & - & - \\
\cline { 2 - 4 } & Wilder Valence & - & 0.3391 \\
\hline \multirow{4}{*}{} & \multicolumn{2}{|c}{} & 0.4569 \\
\hline
\end{tabular}

and valence in MuSe-Wilder. For example, the feature combination "IS13+CNN14+wav2vec+EmoFAN+ResNetFace+DeBERTa" outperforms the best unimodal result by $9.3 \%$ on arousal. However, for MuSe-Sent, the improvement of multimodal fusion is less significant than the best unimodal results, which accords with the findings in the baseline paper [23]. Considering that the discrete sentiment classes of MuSe-Sent come from continuous emotion annotations of MuSe-Wilder, we directly use the best multimodal predictions of MuSe-Wilder as one of the predictions fed into the late fusion model to assist arousal and valence classification in MuSe-Sent. From the last row of Table 3, we can find that only using valence prediction from MuSe-Wilder can significantly improve the performance of valence classification. When integrating arousal prediction, however, the improvement on arousal classification is much smaller. This is reasonable since the best arousal prediction on the test set of MuSe-Wilder is not very good (i.e., only 0.4117 , see Table 4 ). We believe that further improvement on arousal prediction in MuSeWilder will lead to better performance of arousal classification in MuSe-Sent.

\subsection{Submission Results}

The best submission results are shown in Table 4. For MuSe-Wilder, our proposed method achieves CCCs of 0.4117 and 0.6649 for arousal and valence respectively on the test set, which significantly outperforms the baseline system. We notice that our method suffers from the overfitting problem for arousal while generalizes well for valence, which is similar to observations in last year [25]. For MuSe-Sent, we obtain F1-scores of 0.3614 and 0.4451 for arousal and valence, which also outperforms the baseline system, especially for valence. With the encouraging results, we ranked top3 among all participants in these two sub-challenges.

\section{CONCLUSIONS}

In this paper, we present our contributions to the MuSe-Wilder and MuSe-Sent sub-challenges of MuSe 2021. Various handcrafted features and deep representations from three common modalities (i.e., audio, visual, and text) are explored. As for the temporal model, we adopt LSTM as well as the self-attention mechanism to capture
Table 4: The best submission results on the validation set and test set of MUSE-WIDLER and MuSe-Sent.

\begin{tabular}{c|c|c|c|c}
\hline Task & Emotion & Partition & Baseline & Proposed \\
\hline \multirow{4}{*}{ MuSe-Wilder } & Arousal & Val & 0.4929 & $\mathbf{0 . 5 5 4 9}$ \\
& Valence & Val & 0.4863 & $\mathbf{0 . 5 5 6 6}$ \\
& Arousal & Test & 0.3386 & $\mathbf{0 . 4 1 1 7}$ \\
& Valence & Test & 0.5974 & $\mathbf{0 . 6 6 4 9}$ \\
\hline \multirow{5}{*}{ MuSe-Sent } & Arousal & Val & 0.3827 & $\mathbf{0 . 4 2 1 4}$ \\
& Valence & Val & 0.3296 & $\mathbf{0 . 4 5 6 9}$ \\
& Arousal & Test & 0.3512 & $\mathbf{0 . 3 6 1 4}$ \\
& Valence & Test & 0.3291 & $\mathbf{0 . 4 4 5 1}$ \\
\hline
\end{tabular}

long-term temporal context. Finally, we use late fusion to exploit complementary information from different modalities. Moreover, we use continuous predictions from MuSe-Wilder to assist discrete classification in MuSe-Sent and we find that the performance of valence classification could be greatly improved. The best-submitted results show that our proposed method achieves significantly better performance than the baseline system in both MuSe-Wilder and MuSe-Sent. Future work includes trying more advanced temporal models and fusion methods, and exploring multi-task learning between MuSe-Wilder and MuSe-Sent.

\section{ACKNOWLEDGMENTS}

This work is supported by the National Key Research \& Development Plan of China (No.2017YFB1002804), the National Natural Science Foundation of China (NSFC) (No.61831022, No.61771472, No.61773379, No.61901473), the Key Program of the Natural Science Foundation of Tianjin (Grant No. 18JCZDJC36300) and the Alibaba Group through Alibaba Innovative Research Program.

\section{REFERENCES}

[1] Samuel Albanie, Arsha Nagrani, Andrea Vedaldi, and Andrew Zisserman. 2018. Emotion recognition in speech using cross-modal transfer in the wild. In Proceedings of the 26th ACM international conference on Multimedia. 292-301.

[2] Alexei Baevski, Henry Zhou, Abdelrahman Mohamed, and Michael Auli. 2020. wav2vec 2.0: A framework for self-supervised learning of speech representations. arXiv preprint arXiv:2006.11477 (2020).

[3] Tadas Baltrusaitis, Amir Zadeh, Yao Chong Lim, and Louis-Philippe Morency. 2018. Openface 2.0: Facial behavior analysis toolkit. In 2018 13th IEEE International Conference on Automatic Face \& Gesture Recognition (FG 2018). IEEE, 59-66.

[4] Emad Barsoum, Cha Zhang, Cristian Canton Ferrer, and Zhengyou Zhang. 2016. Training deep networks for facial expression recognition with crowd-sourced label distribution. In Proceedings of the 18th ACM International Conference on Multimodal Interaction. 279-283.

[5] Kevin Clark, Minh-Thang Luong, Quoc V Le, and Christopher D Manning. 2020. Electra: Pre-training text encoders as discriminators rather than generators. arXiv preprint arXiv:2003.10555 (2020).

[6] Jacob Devlin, Ming-Wei Chang, Kenton Lee, and Kristina Toutanova. 2018. Bert: Pre-training of deep bidirectional transformers for language understanding. arXiv preprint arXiv:1810.04805 (2018)

[7] Florian Eyben, Klaus R Scherer, Björn W Schuller, Johan Sundberg, Elisabeth André, Carlos Busso, Laurence Y Devillers, Julien Epps, Petri Laukka, Shrikanth S Narayanan, et al. 2015. The Geneva minimalistic acoustic parameter set (GeMAPS) for voice research and affective computing. IEEE Transactions on Affective Computing 7, 2 (2015), 190-202.

[8] Florian Eyben, Martin Wöllmer, and Björn Schuller. 2010. Opensmile: the munich versatile and fast open-source audio feature extractor. In Proceedings of the 18th ACM international conference on Multimedia. ACM, 1459-1462.

[9] Jort F Gemmeke, Daniel PW Ellis, Dylan Freedman, Aren Jansen, Wade Lawrence, R Channing Moore, Manoj Plakal, and Marvin Ritter. 2017. Audio set: An ontology and human-labeled dataset for audio events. In 2017 IEEE International Conference on Acoustics, Speech and Signal Processing (ICASSP). IEEE, 776-780. 
[10] Theodoros Giannakopoulos. 2015. pyaudioanalysis: An open-source python library for audio signal analysis. PloS one 10, 12 (2015), e0144610.

[11] Pengcheng He, Xiaodong Liu, Jianfeng Gao, and Weizhu Chen. 2020. Deberta: Decoding-enhanced bert with disentangled attention. arXiv preprint arXiv:2006.03654 (2020)

[12] Shawn Hershey, Sourish Chaudhuri, Daniel PW Ellis, Jort F Gemmeke, Aren Jansen, R Channing Moore, Manoj Plakal, Devin Platt, Rif A Saurous, Bryan Seybold, et al. 2017. CNN architectures for large-scale audio classification. In 2017 IEEE International Conference on Acoustics, Speech and Signal Processing (ICASSP). IEEE, 131-135.

[13] Diederik P Kingma and Jimmy Ba. 2014. Adam: A method for stochastic optimization. arXiv preprint arXiv:1412.6980 (2014).

[14] Qiuqiang Kong, Yin Cao, Turab Iqbal, Yuxuan Wang, Wenwu Wang, and Mark D Plumbley. 2020. Panns: Large-scale pretrained audio neural networks for audio pattern recognition. IEEE/ACM Transactions on Audio, Speech, and Language Processing 28 (2020), 2880-2894.

[15] Zhenzhong Lan, Mingda Chen, Sebastian Goodman, Kevin Gimpel, Piyush Sharma, and Radu Soricut. 2019. Albert: A lite bert for self-supervised learning of language representations. arXiv preprint arXiv:1909.11942 (2019).

[16] I Lawrence and Kuei Lin. 1989. A concordance correlation coefficient to evaluate reproducibility. Biometrics (1989), 255-268.

[17] Yinhan Liu, Myle Ott, Naman Goyal, Jingfei Du, Mandar Joshi, Danqi Chen, Omer Levy, Mike Lewis, Luke Zettlemoyer, and Veselin Stoyanov. 2019. Roberta: A robustly optimized bert pretraining approach. arXiv preprint arXiv:1907.11692 (2019).

[18] Ali Mollahosseini, Behzad Hasani, and Mohammad H Mahoor. 2017. Affectnet: A database for facial expression, valence, and arousal computing in the wild. IEEE Transactions on Affective Computing 10, 1 (2017), 18-31.

[19] Vassil Panayotov, Guoguo Chen, Daniel Povey, and Sanjeev Khudanpur. 2015. Librispeech: an asr corpus based on public domain audio books. In 2015 IEEE international conference on acoustics, speech and signal processing (ICASSP). IEEE, 5206-5210.

[20] Omkar M Parkhi, Andrea Vedaldi, and Andrew Zisserman. 2015. Deep face recognition. (2015).
[21] Adam Paszke, Sam Gross, Francisco Massa, Adam Lerer, James Bradbury, Gregory Chanan, Trevor Killeen, Zeming Lin, Natalia Gimelshein, Luca Antiga, et al. 2019. Pytorch: An imperative style, high-performance deep learning library. In Advances in neural information processing systems. 8026-8037.

[22] Björn Schuller, Stefan Steidl, Anton Batliner, Alessandro Vinciarelli, Klaus Scherer, Fabien Ringeval, Mohamed Chetouani, Felix Weninger, Florian Eyben, Erik Marchi, et al. 2013. The INTERSPEECH 2013 computational paralinguistics challenge: Social signals, conflict, emotion, autism. In Proceedings INTERSPEECH 2013, 14th Annual Conference of the International Speech Communication Association, Lyon, France.

[23] Lukas Stappen, Alice Baird, Lukas Christ, Lea Schumann, Benjamin Sertolli, Eva-Maria Messner, Erik Cambria, Guoying Zhao, and Björn W Schuller. 2021. The MuSe 2021 Multimodal Sentiment Analysis Challenge: Sentiment, Emotion, Physiological-Emotion, and Stress. In Proceedings of the 2nd International on Multimodal Sentiment Analysis Challenge and Workshop (Chengdu, China). Association for Computing Machinery, New York, NY, USA.

[24] Lukas Stappen, Alice Baird, Lea Schumann, and Björn Schuller. 2021. The Multimodal Sentiment Analysis in Car Reviews (MuSe-CaR) Dataset: Collection, Insights and Improvements. IEEE Transactions on Affective Computing -, - (2021),

[25] Licai Sun, Zheng Lian, Jianhua Tao, Bin Liu, and Mingyue Niu. 2020. Multi-modal continuous dimensional emotion recognition using recurrent neural network and self-attention mechanism. In Proceedings of the 1st International on Multimodal Sentiment Analysis in Real-life Media Challenge and Workshop. 27-34.

[26] Antoine Toisoul, Jean Kossaifi, Adrian Bulat, Georgios Tzimiropoulos, and Maja Pantic. 2021. Estimation of continuous valence and arousal levels from faces in naturalistic conditions. Nature Machine Intelligence 3, 1 (2021), 42-50.

[27] Ashish Vaswani, Noam Shazeer, Niki Parmar, Jakob Uszkoreit, Llion Jones, Aidan N Gomez, Łukasz Kaiser, and Illia Polosukhin. 2017. Attention is all you need. In Advances in neural information processing systems. 5998-6008.

[28] Sicheng Zhao, Shangfei Wang, Mohammad Soleymani, Dhiraj Joshi, and Qiang Ji. 2019. Affective computing for large-scale heterogeneous multimedia data: A survey. ACM Transactions on Multimedia Computing, Communications, and Applications (TOMM) 15, 3s (2019), 1-32. 